\title{
Time-varying LQG control for vibration of coupled vehicle-bridge system
}

\author{
G. SONG \\ State Key Laboratory of Bridge Structural Dynamics, \\ China Merchants Chongqing Communications Research\&Design Institute Co., Ltd., Chongqing, P.R.China \\ e-mail: songgang2@tom.com
}

\begin{abstract}
Time-varying linear quadratic Gaussian (LQG) control for vibration of coupled vehicle-bridge system is studied. The vehicle is modeled as a moving mass model with three degrees of freedom, which consists of vehicle body, bogie and wheel. Active suspensions are adopted for the primary and secondary ones, and the control forces are produced by two actuators placed between the bogie and wheel, and between the vehicle body and the bogie, respectively. Vehicle-bridge coupling systems are time-dependent, which lead to the timevarying Riccati differential equation and the time-varying Kalman-Bucy filter equation in the LQG controller design. However, both of them are solved precisely via precise integration method and symplectic conservative perturbation method. In the example, the time history responses of the bridge and the vehicle were calculated respectively for the vehicle with passive suspensions or with active suspensions. Numerical results show that with active suspensions adopted, ride comfort can be improved when the vehicles passing through the bridge.
\end{abstract}

Keywords- vehicle-bridge coupling; time-varying; LQG control; precise integration; symplectic conservative perturbation

\section{INTRODUCTION}

Developments in design technology and material qualities in civil engineering enable the construction of more light and slender bridges. Vibration suppression for coupled vehicle-bridge system has received considerable attention from researchers in the past years. For the sake of safety and serviceability of bridges and better riding comfort or maneuverability of passing vehicles, the dynamic responses of bridges and moving vehicles should be kept within allowable limits. Kwon et al. [1] studied the moving load induced vibration control of bridges using tuned mass dampers when TGV high-speed trains were modeled as 2 degree of freedom systems consisting of the wheel and body. Yau and Yang [2] developed a wideband multiple tuned mass dampers (MTMD) system for reducing the multiple resonant responses of continuous truss bridges to moving train loads, and demonstrated the robustness of the proposed method in vibration reduction. Wang et al. [3] treated a train as series of moving forces, moving masses or moving suspension masses and investigated the influence of various vehicle models on the bridge features with or without passive tuned mass dampers.

This paper presents the applicability of time-varying linear quadratic Gaussian (LQG) control to suppress vibration of the coupled vehicle-bridge system. Section 2 deals with the formulation procedures for the equations of motion of the bridges under moving vehicles. Section 3 deals with the procedures of time-varying LQG controller design, and Section 4 deals with the simulation of such control problem. Section 5 details the numerical analysis and Section 6 shows the conclusions.

\section{MODEL BUILDING}

In Fig. 1, a model is presented for a simply supported girder bridge under a moving rail carriage. Only vertical vibration of the carriage and bridge is considered. The carriage is modeled as a moving mass model with three degrees of freedom, which consists of vehicle body, bogie and wheel and each of the suspension systems is represented by a spring-dashpot unit. The bridge considered is idealized as a uniform continuous Bernoulli-Euler beam. In Fig. 1, $m_{b}$, $m_{t}$ and $m_{w}$ are the mass of vehicle body, bogie and wheel respectively; $k_{b}, c_{b}, k_{t}$ and $c_{t}$ are the spring stiffnesses and damping coefficients of the secondary and primary suspension systems respectively; $z_{b}, z_{t}$ and $z_{w}$ are the vertical displacements of the vehicle body, bogie and wheel respectively; $v$ is the constant velocity of carriage; the wheel is assumed to contact continuously with the rails as it rolls over and $r$ is the vertical track irregularities under the wheel. Nonlinear Hertzian theory is usually used to model the wheel-rail contact [4], but in this paper the contact force between the rail and wheel is approximated to be generated by a spring and $k_{w}$ is the equivalent spring stiffness. The length of bridge is denoted as $l$, and its vertical flexural stiffness, viscous damping coefficient and mass per unit length are denoted as EI, $c$ and $m$ respectively. Vertical deformation of the bridge is $z$. Control forces $u_{1}$ and $u_{2}$ are produced by two actuators placed between the bogie and wheel, and between the vehicle body and bogie respectively. Dynamics of the actuators is neglected. The carriage is assumed to be initial static on the roadbed and with a distance $l_{r}$ to the left end of the bridge. Irregularities of the track lying on the roadbed satisfy the same statistic properties as those on the bridge and stiffness of the roadbed is assumed to be infinite. 


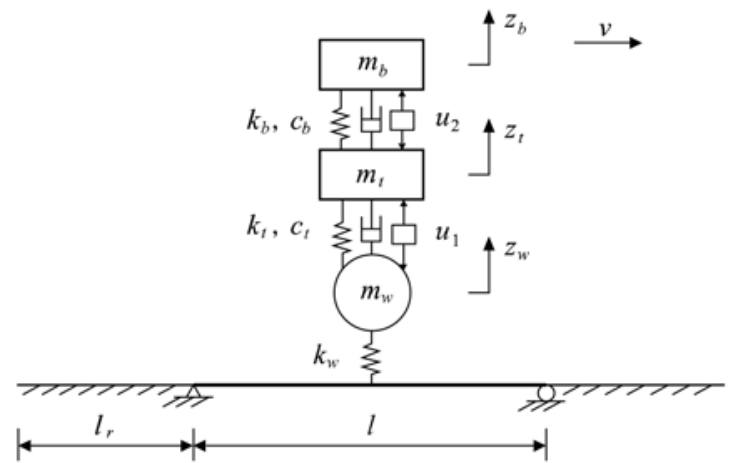

Figure 1. A rail carriage traveling on a simply supported girder bridge.

The equations of motion for the carriage can be written as

$$
\begin{gathered}
m_{w} \ddot{z}_{w}-f_{0}+f_{1}=0 \\
m_{t} \ddot{z}_{t}-f_{1}+f_{2}=0 \\
m_{b} \ddot{z}_{b}-f_{2}=0
\end{gathered}
$$

in which

$$
\begin{gathered}
f_{0}=-k_{w}\left[z_{w}-z(\xi, t) D(t)-r\right] \\
f_{1}=-c_{t}\left[\dot{z}_{t}-\dot{z}_{w}\right]-k_{t}\left[z_{t}-z_{w}\right]+u_{1} \\
f_{2}=-c_{b}\left(\dot{z}_{b}-\dot{z}_{t}\right)-k_{b}\left(z_{b}-z_{t}\right)+u_{2}
\end{gathered}
$$

$\xi$ is the carriage location on the bridge at time $t$ and can be described as $\xi=v t-l_{r}$. Coefficient $D(t)$ depends on the interval of the motion defined as

$$
D(t)= \begin{cases}1, & l_{r} / v \leq t \leq\left(l_{r}+l\right) / v \\ 0, & t<l_{r} / v \text { or } t>\left(l_{r}+l\right) / v\end{cases}
$$
as [5]

The equation of motion of the bridge can be expressed

$$
\begin{aligned}
& E I \frac{\partial^{4} z(x, t)}{\partial x^{4}}+m \frac{\partial^{2} z(x, t)}{\partial t^{2}}+c \frac{\partial z(x, t)}{\partial t} \\
& =-\left[f_{0}+\left(m_{b}+m_{t}+m_{w}\right) g\right] D(t) \delta(x-\xi)
\end{aligned}
$$

where $x$ denotes the distance between the dynamic location to the left end of the bridge; and $\delta$ is the Dirac delta function. By separation of variables, the vertical deformation of the bridge $z(x, t)$ can be expressed as

$$
z(x, t)=\sum_{i=1}^{n} \phi_{i}(x) q_{i}(t)
$$

in which $\phi_{i}(x)(i=1,2, \ldots, n)$ is the vibration mode and $q_{i}(t)(i=1,2, \ldots, n)$ is the generalized coordinates. $\phi_{i}(x)$ can be selected as

$$
\phi_{i}(x)=\sin \left(\frac{i \pi x}{l}\right)
$$

Substitute (9) into (4) and (8), and notice

$$
\begin{aligned}
& \int_{0}^{l}\left[f_{0}+\left(m_{b}+m_{t}+m_{w}\right) g\right] D(t) \delta(x-\xi) \phi_{i}(x) d x \\
& =\left[f_{0}+\left(m_{b}+m_{t}+m_{w}\right) g\right] D(t) \phi_{i}(\xi) \\
& \int_{0}^{l} \phi_{i}(x) \phi_{j}(x) d x=\left\{\begin{array}{cl}
l / 2 & \text { for } i=j \\
0 & \text { for } i \neq j
\end{array}\right.
\end{aligned}
$$

the following equations can be obtained:

$$
\begin{aligned}
& f_{0}=-k_{w}\left[z_{w}-\sum_{j=1}^{n} \sin \left(\frac{j \pi \xi}{l}\right) q_{j}(t) D(t)-r\right] \\
& m \frac{l}{2} \ddot{q}_{i}(t)+c \frac{l}{2} \dot{q}_{i}(t)+\frac{l}{2} \frac{i^{4} \pi^{4}}{l^{4}} E I q_{i}(t) \\
& =-\left[f_{0}+\left(m_{b}+m_{t}+m_{w}\right) g\right] D(t) \sin \left(\frac{i \pi \xi}{l}\right), i=1,2, \ldots, n
\end{aligned}
$$

Equations (1), (2), (3), (5), (6), (13) and (14) can be written in matrix form as

$$
\dot{\boldsymbol{x}}=\boldsymbol{A}(t) \boldsymbol{x}+\boldsymbol{B} \boldsymbol{u}+\boldsymbol{F}_{x}(t)+\boldsymbol{H} \dot{r}
$$

where

$$
\begin{gathered}
\boldsymbol{x}=\left\{\begin{array}{lllllll}
\boldsymbol{x}_{q 1}^{\mathrm{T}} & \boldsymbol{x}_{q 2}^{\mathrm{T}}
\end{array}\right\}^{\mathrm{T}}, \quad \boldsymbol{u}=\left\{\begin{array}{lllll}
u_{1} & u_{2}
\end{array}\right\}^{\mathrm{T}} \\
\boldsymbol{x}_{q 1}=\left\{\begin{array}{llllllll}
q_{1} & q_{2} & \cdots & q_{n} & z_{b}-z_{t} & z_{t}-z_{w} & z_{w}-r
\end{array}\right\}^{\mathrm{T}} \\
\boldsymbol{x}_{q 2}=\left\{\begin{array}{llllllll}
\dot{q}_{1} & \dot{q}_{2} & \cdots & \dot{q}_{n} & \dot{z}_{b} & \dot{z}_{t} & \dot{z}_{w}
\end{array}\right\}^{\mathrm{T}}
\end{gathered}
$$

In this study, the vertical track irregularity is assumed to be of random nature and characterized by the following power spectral density (PSD) function $S_{r}(\Omega)[4,5]$ :

$$
S_{r}(\Omega)=\frac{A_{v} \Omega_{\mathrm{c}}^{2}}{\left(\Omega^{2}+\Omega_{r}^{2}\right)\left(\Omega^{2}+\Omega_{c}^{2}\right)}
$$

where $\Omega$ denotes the spatial frequency $(\mathrm{rad} / \mathrm{m})$ and $A_{v}$ (m), $\Omega_{r}(\mathrm{rad} / \mathrm{m})$ and $\Omega_{c}(\mathrm{rad} / \mathrm{m})$ are relevant parameters. When the carriage has constant velocity $v(\mathrm{~m} / \mathrm{s})$, the temporal PSD of (16) can be written in terms of the angular frequency $\omega(\mathrm{rad} / \mathrm{s}), \omega=v \Omega$, as

$$
S_{r}(\omega)=\frac{A_{v} v^{3} \Omega_{c}^{2}}{\left(\omega^{2}+v^{2} \Omega_{r}^{2}\right)\left(\omega^{2}+v^{2} \Omega_{c}^{2}\right)}
$$

Equation (17) means that $\dot{r}$ can be generated by passing a white noise process through a linear filter given as

$$
\dot{r}=A_{c} \boldsymbol{\Gamma}, \quad \dot{\boldsymbol{\Gamma}}=\boldsymbol{B}_{c} \boldsymbol{\Gamma}+\boldsymbol{D}_{c} \eta_{1}
$$

in which $\eta_{1}$ is the zero-mean Gaussian white noise with intensity $W_{1}=\pi$ and

$$
\begin{gathered}
\boldsymbol{A}_{c}=\left[\begin{array}{ll}
1 & 0
\end{array}\right], \quad \boldsymbol{B}_{c}=\left[\begin{array}{cc}
0 & 1 \\
-v^{2} \Omega_{c} \Omega_{r} & -v\left(\Omega_{c}+\Omega_{r}\right)
\end{array}\right] \\
\boldsymbol{D}_{c}=\left\{\begin{array}{c}
v \Omega_{c} \sqrt{A_{v} v} \\
-v^{2} \Omega_{c}\left(\Omega_{c}+\Omega_{r}\right) \sqrt{A_{v} v}
\end{array}\right\}
\end{gathered}
$$

For the vehicle-bridge coupled system control problem, it is impossible to measure all state variables. Assuming that only the vertical accelerations of the vehicle body and the relative displacements of the secondary and primary suspensions can be measured by accelerometers and relative displacement sensors, and assuming that measurement noise always exists, gives

$$
y_{1}=\ddot{z}_{b}+v_{1}, \quad y_{2}=z_{b}-z_{t}+v_{2}, y_{3}=z_{t}-z_{w}+v_{3}
$$

where $v_{1}, v_{2}$ and $v_{3}$ are measurement noises, which can be assumed to be zero-mean Gaussian white and be uncorrelated with each other and with the track input. Equation (19) can be expressed in state vector form as

$$
\boldsymbol{y}=\boldsymbol{C} \boldsymbol{x}+\boldsymbol{D u}+\boldsymbol{w}_{2}
$$


where

$$
\boldsymbol{y}=\left[\begin{array}{lll}
y_{1} & y_{2} & y_{3}
\end{array}\right]^{\mathrm{T}}, \quad \boldsymbol{w}_{2}=\left[\begin{array}{lll}
v_{1} & v_{2} & v_{3}
\end{array}\right]^{\mathrm{T}}
$$

$\boldsymbol{w}_{2}$ is the measurement noise vector with

$$
\begin{gathered}
E\left[\boldsymbol{w}_{2}\right]=\mathbf{0}, E\left[\boldsymbol{w}_{2}\left(t_{1}\right) \eta_{1}\left(t_{2}\right)\right]=\mathbf{0} \\
E\left[\boldsymbol{w}_{2}\left(t_{1}\right) \boldsymbol{w}_{2}^{\mathrm{T}}\left(t_{2}\right)\right]=\boldsymbol{W}_{2} \delta\left(t_{2}-t_{1}\right)
\end{gathered}
$$

where $t_{1}$ and $t_{2}$ are any two times, $E[]$ is the mean value of [ ], $\delta(t)$ is the Dirac delta function and $\boldsymbol{W}_{2}$ is the intensity matrix of measurement noises.

\section{LQG CONTROLLER DESIGN}

In this study, the purpose of the active suspension is to decrease the vehicle body acceleration, which is needed in order to improve the ride comfort of passengers. Additionally, for practical reason, both excessive suspension working space requirements and the need for excessive control forces should be avoided. Thus the performance index to be minimized can be written as

$$
\begin{aligned}
J= & E\left\{\frac { 1 } { 2 } \int _ { 0 } ^ { t _ { f } } \left[\rho_{1} \ddot{z}_{b}^{2}+\rho_{2}\left(z_{b}-z_{t}\right)^{2}+\rho_{3}\left(z_{t}-z_{w}\right)^{2}+\rho_{4} u_{1}^{2}\right.\right. \\
& \left.\left.+\rho_{5} u_{2}^{2}\right] d t+\frac{1}{2} \boldsymbol{x}^{\mathrm{T}}\left(t_{f}\right) \boldsymbol{S}_{f} \boldsymbol{x}\left(t_{f}\right)\right\}
\end{aligned}
$$

where $\rho_{i}(i=1, \ldots, 5)$ is the weighting coefficient reflecting the designer's preferences; $\boldsymbol{S}_{f}$ is terminal weighting matrix, which is positive semidefinite and symmetric and also determined by designers; $\left[0, t_{f}\right]$ is the control time interval. By expressing the vehicle body acceleration and suspensions relative displacements in terms of the state and input vectors, the performance index of (22) can be written in the quadratic form:

$$
J=E\left\{\frac{1}{2} \int_{0}^{t_{f}}\left[\boldsymbol{x}^{\mathrm{T}} \boldsymbol{Q} \boldsymbol{x}+2 \boldsymbol{x}^{\mathrm{T}} \boldsymbol{N} \boldsymbol{u}+\boldsymbol{u}^{\mathrm{T}} \boldsymbol{R} \boldsymbol{u}\right] d t+\frac{1}{2} \boldsymbol{x}^{\mathrm{T}}\left(t_{f}\right) \boldsymbol{S}_{f} \boldsymbol{x}\left(t_{f}\right)\right\}
$$

where $\boldsymbol{Q}$ and $\boldsymbol{R}$ are symmetric matrices such that $\boldsymbol{R}>\mathbf{0}$, $\boldsymbol{Q}_{n 1}=\boldsymbol{Q}-\boldsymbol{N} \boldsymbol{R}^{-1} \boldsymbol{N}^{\mathrm{T}} \geq \mathbf{0}$ and $\boldsymbol{N}$ is a constant matrix.

Combining (15) and (18), the system equation with augmented state vector can be written as

$$
\dot{\boldsymbol{x}}_{1}=\boldsymbol{A}_{1}(t) \boldsymbol{x}_{1}+\boldsymbol{B}_{1} \boldsymbol{u}+\boldsymbol{F}_{x 1}(t)+\boldsymbol{H}_{1} \eta_{1}
$$

where

$$
\begin{gathered}
\boldsymbol{x}_{1}=\left\{\begin{array}{l}
\boldsymbol{x} \\
\boldsymbol{\Gamma}
\end{array}\right\}, \boldsymbol{A}_{1}=\left[\begin{array}{cc}
\boldsymbol{A} & \boldsymbol{H} \boldsymbol{A}_{c} \\
\mathbf{0} & \boldsymbol{B}_{c}
\end{array}\right], \boldsymbol{B}_{1}=\left[\begin{array}{c}
\boldsymbol{B} \\
\mathbf{0}
\end{array}\right], \boldsymbol{F}_{x 1}=\left\{\begin{array}{c}
\boldsymbol{F}_{x} \\
\mathbf{0}
\end{array}\right\} \\
\boldsymbol{H}_{1}=\left\{\begin{array}{ll}
\boldsymbol{0} & \boldsymbol{D}_{c}^{\mathrm{T}}
\end{array}\right\}^{\mathrm{T}}
\end{gathered}
$$

Accordingly, measurement equation (20) and the performance index in (23) may be expressed in terms of the augmented state vector as

$$
\begin{gathered}
\boldsymbol{y}=\boldsymbol{C}_{1} \boldsymbol{x}_{1}+\boldsymbol{D} \boldsymbol{u}+\boldsymbol{w}_{2} \\
J=E\left\{\frac{1}{2} \int_{0}^{t_{f}}\left[\boldsymbol{x}_{1}^{\mathrm{T}} \boldsymbol{Q}_{1} \boldsymbol{x}_{1}+2 \boldsymbol{x}_{1}^{\mathrm{T}} \boldsymbol{N}_{1} \boldsymbol{u}+\boldsymbol{u}^{\mathrm{T}} \boldsymbol{R} \boldsymbol{u}\right] d t+\frac{1}{2} \boldsymbol{x}_{1}^{\mathrm{T}}\left(t_{f}\right) \boldsymbol{S}_{1 f} \boldsymbol{x}_{1}\left(t_{f}\right)\right\}
\end{gathered}
$$

where

$$
\boldsymbol{C}_{1}=\left[\begin{array}{ll}
\boldsymbol{C} & \mathbf{0}
\end{array}\right], \quad \boldsymbol{Q}_{1}=\left[\begin{array}{ll}
\boldsymbol{Q} & \mathbf{0} \\
\mathbf{0} & \mathbf{0}
\end{array}\right], \quad \boldsymbol{N}_{1}=\left[\begin{array}{c}
\boldsymbol{N} \\
\mathbf{0}
\end{array}\right], \quad \boldsymbol{S}_{1 f}=\left[\begin{array}{cc}
\boldsymbol{S}_{f} & \mathbf{0} \\
\mathbf{0} & \mathbf{0}
\end{array}\right]
$$

By far, standard forms of the LQG control problem are given by (24)-(26). However, one should note that system matrix $\boldsymbol{A}_{1}$ is time-dependent. It has been proved that the separation principle holds for the LQG control problem and so its solution is the combination of a deterministic optimal state feedback regulator and a stochastic optimal estimator [6]. The optimal control forces can be given by

$$
\boldsymbol{u}(t)=-\boldsymbol{K}_{c}(t) \hat{\boldsymbol{x}}_{1}(t)
$$

where $\hat{\boldsymbol{x}}_{1}$ is the estimate of $\boldsymbol{x}_{1}$ and $\boldsymbol{K}_{c}$ is the gain matrix for the optimal regulator, such as

$$
\boldsymbol{K}_{c}(t)=\boldsymbol{R}^{-1}\left[\boldsymbol{B}_{1}^{\mathrm{T}} \boldsymbol{S}(t)+\boldsymbol{N}_{1}^{\mathrm{T}}\right]
$$

$\boldsymbol{S}$ satisfies the Riccati equation

$$
\dot{\boldsymbol{S}}=-\boldsymbol{S} \boldsymbol{A}_{n}(t)-\boldsymbol{A}_{n}^{\mathrm{T}}(t) \boldsymbol{S}+\boldsymbol{S} \boldsymbol{B}_{1} \boldsymbol{R}^{-1} \boldsymbol{B}_{1}^{\mathrm{T}} \boldsymbol{S}-\boldsymbol{Q}_{n}, \boldsymbol{S}\left(t_{f}\right)=\boldsymbol{S}_{1 f}
$$

in which

$$
\boldsymbol{A}_{n}(t)=\boldsymbol{A}_{1}(t)-\boldsymbol{B}_{1} \boldsymbol{R}^{-1} \boldsymbol{N}_{1}^{\mathrm{T}}, \quad \boldsymbol{Q}_{n}=\boldsymbol{Q}_{1}-\boldsymbol{N}_{1} \boldsymbol{R}^{-1} \boldsymbol{N}_{1}^{\mathrm{T}}
$$

and $\hat{\boldsymbol{x}}_{1}$ can be obtained from the Kalman-Bucy filter, i.e.

$\dot{\hat{\boldsymbol{x}}}_{1}=\boldsymbol{A}_{1}(t) \hat{\boldsymbol{x}}_{1}+\boldsymbol{B}_{1} \boldsymbol{u}+\boldsymbol{F}_{x 1}(t)+\boldsymbol{K}_{f}(t)\left[\boldsymbol{y}-\boldsymbol{C}_{1} \hat{\boldsymbol{x}}_{1}-\boldsymbol{D} \boldsymbol{u}\right], \hat{\boldsymbol{x}}_{1}(0)=\hat{\boldsymbol{x}}_{10}$

Here the optimal estimator gain $\boldsymbol{K}_{f}$ is given by

$$
\boldsymbol{K}_{f}(t)=\boldsymbol{P}(t) \boldsymbol{C}_{1}^{\mathrm{T}} \boldsymbol{W}_{2}^{-1}
$$

and can be obtained from another Riccati equation:

$$
\dot{\boldsymbol{P}}=\boldsymbol{A}_{1}(t) \boldsymbol{P}+\boldsymbol{P} \boldsymbol{A}_{1}^{\mathrm{T}}(t)-\boldsymbol{P} \boldsymbol{C}_{1}^{\mathrm{T}} \boldsymbol{W}_{2}^{-1} \boldsymbol{C}_{1} \boldsymbol{P}+\boldsymbol{H}_{1} W_{1} \boldsymbol{H}_{1}^{\mathrm{T}}, \quad \boldsymbol{P}(0)=\boldsymbol{P}_{0}
$$

In this study, the carriage and bridge are assumed to be initially static and $\hat{\boldsymbol{x}}_{10}$ and $\boldsymbol{P}_{0}$ can be both regarded as null. Note that (29) and (32) give the time-varying Riccati differential equations, not the usual algebraic ones. Such Riccati differential equations are usually difficult to solve [7, 8 ], but are easily dealt with by adopting the precise integration method and symplectic conservative perturbation method [9, 10]. Note that $\boldsymbol{S}, \boldsymbol{K}_{c}, \boldsymbol{P}$ and $\boldsymbol{K}_{f}$ can all be calculated off-line since they are independent of the measurements. Substituting (27) into (30) gives

$$
\dot{\hat{\boldsymbol{x}}}_{1}=\left[\boldsymbol{A}_{1}(t)-\boldsymbol{B}_{1} \boldsymbol{K}_{c}(t)\right] \hat{\boldsymbol{x}}_{1}+\boldsymbol{F}_{x 1}(t)+\boldsymbol{K}_{f}(t)\left[\boldsymbol{y}-\boldsymbol{C}_{1} \hat{\boldsymbol{x}}_{1}+\boldsymbol{D} \boldsymbol{K}_{c}(t) \hat{\boldsymbol{x}}_{1}\right]
$$

Denote adjacent sampling times as $t_{k}, t_{k+1}(k=0,1,2, \ldots)$. By using Duhamel integration, the solution of (33) can be found in the following form [11]:

$$
\begin{aligned}
\hat{\boldsymbol{x}}_{1}\left(t_{k+1}\right) & =\boldsymbol{\Phi}\left(t_{k+1}, t_{k}\right) \hat{\boldsymbol{x}}_{1}\left(t_{k}\right)+\int_{t_{k}}^{t_{k+1}} \boldsymbol{\Phi}\left(t_{k+1}, t\right)\left\{\boldsymbol{F}_{x 1}(t)\right. \\
& \left.+\boldsymbol{K}_{f}(t)\left[\boldsymbol{y}(t)-\boldsymbol{C}_{1} \hat{\boldsymbol{x}}_{1}(t)+\boldsymbol{D} \boldsymbol{K}_{c}(t) \hat{\boldsymbol{x}}_{1}(t)\right]\right\} d t
\end{aligned}
$$

in which $\boldsymbol{\Phi}$ is the state transfer matrix. Evaluating the integral via the trapezoidal rule yields

$$
\begin{aligned}
\hat{\boldsymbol{x}}_{1}\left(t_{k+1}\right)= & \boldsymbol{\Phi}\left(t_{k+1}, t_{k}\right) \hat{\boldsymbol{x}}_{1}\left(t_{k}\right)+\frac{\mathbf{1}}{\mathbf{2}} \boldsymbol{\Phi}\left(t_{k+1}, t_{k}\right)\left\{\boldsymbol{F}_{x 1}\left(t_{k}\right)+\boldsymbol{K}_{f}\left(t_{k}\right)\left[\boldsymbol{y}\left(t_{k}\right)\right.\right. \\
& \left.\left.-\boldsymbol{C}_{1} \hat{\boldsymbol{x}}_{1}\left(t_{k}\right)+\boldsymbol{D} \boldsymbol{K}_{c}\left(t_{k}\right) \hat{\boldsymbol{x}}_{1}\left(t_{k}\right)\right]\right\} \Delta t+\frac{1}{2}\left\{\boldsymbol{F}_{x 1}\left(t_{k+1}\right)\right. \\
& \left.+\boldsymbol{K}_{f}\left(t_{k+1}\right)\left[\boldsymbol{y}\left(t_{k+1}\right)-\boldsymbol{C}_{1} \hat{\boldsymbol{x}}_{1}\left(t_{k+1}\right)+\boldsymbol{D} \boldsymbol{K}_{c}\left(t_{k+1}\right) \hat{\boldsymbol{x}}_{1}\left(t_{k+1}\right)\right]\right\} \Delta t
\end{aligned}
$$

in which $\Delta t=t_{k+1}-t_{k}$. Equation (35) can be rewritten as 


$$
\hat{\boldsymbol{x}}_{1}\left(t_{k+1}\right)=\left(\boldsymbol{I}+\boldsymbol{Y}_{k}\right)^{-1}\left[\boldsymbol{\Phi}\left(t_{k+1}, t_{k}\right) \boldsymbol{p}_{k 1}+\boldsymbol{p}_{k 2}\right]
$$

where

$$
\begin{aligned}
\boldsymbol{Y}_{k}= & \boldsymbol{K}_{f}\left(t_{k+1}\right)\left[\boldsymbol{C}_{1}-\boldsymbol{D} \boldsymbol{K}_{c}\left(t_{k+1}\right)\right] \Delta t / 2 \\
\boldsymbol{p}_{k 1}= & \hat{\boldsymbol{x}}_{1}\left(t_{k}\right)+\boldsymbol{F}_{x 1}\left(t_{k}\right) \Delta t / 2+\boldsymbol{K}_{f}\left(t_{k}\right)\left[\boldsymbol{y}\left(t_{k}\right)-\boldsymbol{C}_{1} \hat{\boldsymbol{x}}_{1}\left(t_{k}\right)\right. \\
& \left.+\boldsymbol{D} \boldsymbol{K}_{c}\left(t_{k}\right) \hat{\boldsymbol{x}}_{1}\left(t_{k}\right)\right] \Delta t / 2 \\
\boldsymbol{p}_{k 2}= & {\left[\boldsymbol{F}_{x 1}\left(t_{k+1}\right)+\boldsymbol{K}_{f}\left(t_{k+1}\right) \boldsymbol{y}\left(t_{k+1}\right)\right] \Delta t / 2 }
\end{aligned}
$$

At time $t_{k+1}, \boldsymbol{y}\left(t_{k}\right)$ and $\boldsymbol{y}\left(t_{k+1}\right)$ have been measured, which means that $\boldsymbol{p}_{k 1}$ and $\boldsymbol{p}_{k 2}$ are known and so $\hat{\boldsymbol{x}}_{1}\left(t_{k+1}\right)$ can be obtained from (36). The control force vector $\boldsymbol{u}$ needed at time $t_{k+1}$ can then be obtained from (27). Note that both $\boldsymbol{Y}_{k}$ and $\boldsymbol{\Phi}\left(t_{k+1}, t_{k}\right)$ can be calculated off-line. The method to obtain $\boldsymbol{\Phi}\left(t_{k+1}, t_{k}\right)$ can be found in $[9,11]$.

\section{SIMULATION OF THE TIME-VARYING LQG CONTROL PROBLEM}

Carriage and bridge responses can be simulated by combining (24), (25), (27) and (30) into

$$
\dot{\boldsymbol{q}}=\hat{\boldsymbol{A}}(t) \boldsymbol{q}+\hat{\boldsymbol{f}}(t)
$$

where

$$
\begin{gathered}
\hat{\boldsymbol{A}}(t)=\left[\begin{array}{cc}
\boldsymbol{A}_{1}(t) & -\boldsymbol{B}_{1} \boldsymbol{K}_{c}(t) \\
\boldsymbol{K}_{f}(t) \boldsymbol{C}_{1} & \boldsymbol{A}_{1}(t)-\boldsymbol{B}_{1} \boldsymbol{K}_{c}(t)-\boldsymbol{K}_{f}(t) \boldsymbol{C}_{1}
\end{array}\right] \\
\hat{\boldsymbol{f}}(t)=\left\{\begin{array}{c}
\boldsymbol{F}_{x 1}(t)+\boldsymbol{H}_{1} \eta_{1}(t) \\
\boldsymbol{F}_{x 1}(t)+\boldsymbol{K}_{f}(t) \boldsymbol{w}_{2}(t)
\end{array}\right\}, \quad \boldsymbol{q}=\left\{\begin{array}{l}
\boldsymbol{x}_{1} \\
\hat{\boldsymbol{x}}_{1}
\end{array}\right\}
\end{gathered}
$$

In each step $\left[t_{k}, t_{k}+\Delta t\right]$, assuming that $\hat{\boldsymbol{A}}$ keeps constant and $\hat{\boldsymbol{f}}$ varies linearly gives

$$
\begin{aligned}
\boldsymbol{q}\left(t_{k}+\Delta t\right) & =\exp \left(\hat{\boldsymbol{A}}\left(t_{k}\right) \Delta t\right)\left\{\boldsymbol{q}\left(t_{k}\right)+\hat{\boldsymbol{A}}^{-1}\left(t_{k}\right)\left[\boldsymbol{r}_{0}+\hat{\boldsymbol{A}}^{-1}\left(t_{k}\right) \boldsymbol{r}_{1}\right]\right\} \\
& -\hat{\boldsymbol{A}}^{-1}\left(t_{k}\right)\left[\boldsymbol{r}_{0}+\hat{\boldsymbol{A}}^{-1}\left(t_{k}\right) \boldsymbol{r}_{1}+\boldsymbol{r}_{1} \Delta t\right]
\end{aligned}
$$

in which

$$
\boldsymbol{r}_{0}=\hat{\boldsymbol{f}}\left(t_{k}\right), \quad \boldsymbol{r}_{1}=\left[\hat{\boldsymbol{f}}\left(t_{k}+\Delta t\right)-\hat{\boldsymbol{f}}\left(t_{k}\right)\right] / \Delta t
$$

Precise solution of $\exp \left[\hat{\boldsymbol{A}}\left(t_{k}\right) \Delta t\right]$ can be found in [12].

\section{NUMERICAL RESULTS}

The proposed numerical method is applied to the coupled vehicle-bridge system of Fig. 1 with passive suspension and time-varying LQG controlled active one compared. The values used for the carriage parameters were: $m_{b}=14800 \mathrm{~kg}, m_{t}=1700 \mathrm{~kg}, m_{w}=3800 \mathrm{~kg}, k_{b}=820 \times 10^{3} \mathrm{~N} / \mathrm{m}$, $c_{b}=217.4 \times 10^{3} \mathrm{~N} \mathrm{~s} / \mathrm{m}, k_{t}=3492 \times 10^{3} \mathrm{~N} / \mathrm{m}, c_{t}=120 \times 10^{3} \mathrm{~N} \mathrm{~s} / \mathrm{m}$. The value used for the equivalent wheel-track contact stiffness $k_{w}$ was $1705352009 \mathrm{~N} / \mathrm{m}$ and the carriage was assumed to be moving in a straight line with constant velocity $v=22.22 \mathrm{~m} / \mathrm{s}$. The values used for the bridge parameters were: $E I=5.0248 \times 10^{10} \mathrm{~N} \mathrm{~m}^{2}, m=4965 \mathrm{~kg} / \mathrm{m}$, $c=6089.462 \mathrm{~N} \mathrm{~s} / \mathrm{m}, l=45 \mathrm{~m}$. The number of vibration modes $n$ in (9) was selected as 10 . The carriage and bridge are initially static and the initial location of the carriage to the left end of bridge $l_{r}$ was selected as $45 \mathrm{~m}$. The whole running distance of the carriage was $l_{s}=l_{r}+l$ and the control time interval was selected as the whole running time of the carriage with $t_{f}=l_{s} / v$. The values of the track irregularity PSD parameters in (16) were: $A_{v}=2.39 \times 10^{-5} \mathrm{~m}$, $\Omega_{r}=2.06 \times 10^{-2} \mathrm{rad} / \mathrm{m}, \Omega_{c}=0.825 \mathrm{rad} / \mathrm{m}$. The intensity matrix of the measurement noise vector was assumed to be

$$
\boldsymbol{W}_{2}=\operatorname{diag}\left(1 \times 10^{-4}, 1 \times 10^{-6}, 1 \times 10^{-6}\right)
$$

The weighting constants in the performance index of (22) were selected as $\rho_{1}=10, \rho_{2}=1 \times 10^{6}, \rho_{3}=1, \rho_{4}=1 \times 10^{-7}$, $\rho_{5}=1 \times 10^{-6}$ and the terminal weighting matrix $\boldsymbol{S}_{f}$ was selected as

$$
\boldsymbol{S}_{f}=\operatorname{diag}\left(10^{5} \boldsymbol{I}_{n}, \quad 10^{4} \boldsymbol{I}_{3}, \quad 10^{2} \boldsymbol{I}_{n}, \quad 10 \boldsymbol{I}_{3}\right)
$$

The history responses of vertical body acceleration and relative displacement of secondary suspension are shown in Fig. 2, for both the passive (i.e. uncontrolled) and active suspension (i.e. controlled) cases. Fig. 3 depicts the history responses of control forces of active suspension. Peak values of the carriage and bridge responses in the whole running process are shown in Table I. It can be seen that peak response of the vehicle body acceleration was decreased for the controlled case, i.e. from $2.43 \mathrm{~m} \mathrm{~s}^{-2}$ to $2.18 \mathrm{~m} \mathrm{~s}^{-2}$, with a reduction of $10 \%$; and the peak values of the relative displacement of secondary suspension also got a large reduction, i.e. from $13.98 \mathrm{~mm}$ to $11.97 \mathrm{~mm}$, with a reduction of $14 \%$. Meanwhile, excessive increase of the bridge deflection responses was successful to be avoided. Note that the relative displacement of the primary suspension had a large increase, i.e. from $8.82 \mathrm{~mm}$ to $11.50 \mathrm{~mm}$, with a increase of $30 \%$; however, such a suspension relative displacement response, i.e. peak value is $11.50 \mathrm{~mm}$, in most cases still can be accepted for the vehicle design.
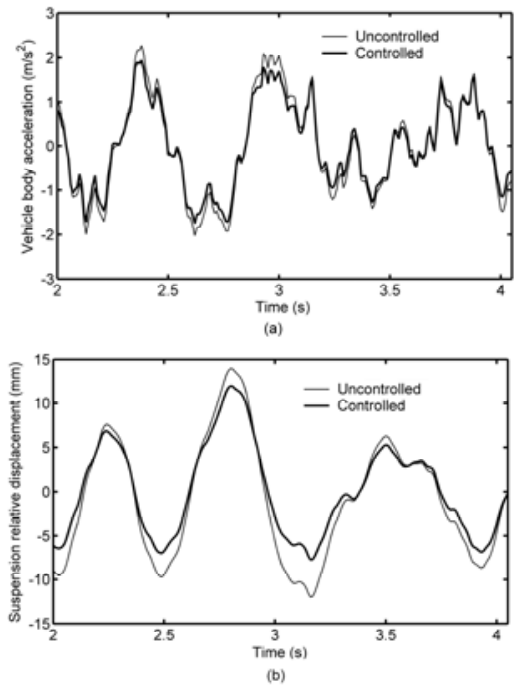

Figure 2. Responses of vertical vehicle body acceleration and secondary suspension relative displacement. 

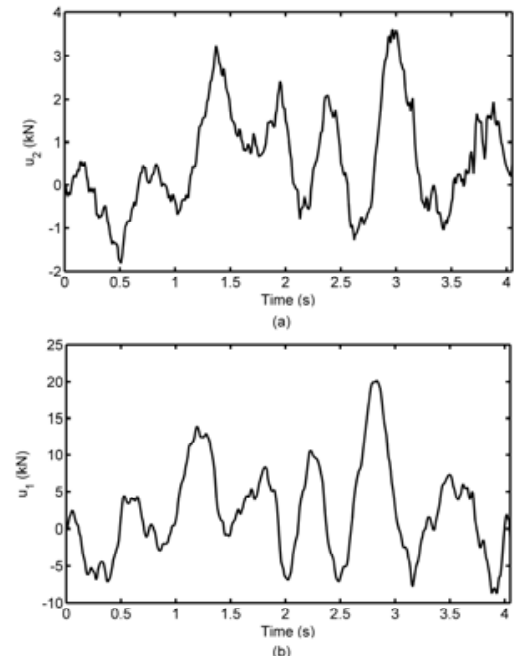

Figure 3. Responses of control forces $u_{2}$ and $u_{1}$.

Table I. Peak values of the carriage and bridge responses

\begin{tabular}{cccc}
\hline Peak value & Uncontrolled & Controlled & Reduction \\
\hline$\ddot{z}_{b}\left(\mathrm{~m} \mathrm{~s}^{-2}\right)$ & 2.43 & 2.18 & $10 \%$ \\
$z_{b}-z_{t}(\mathrm{~mm})$ & 13.98 & 11.97 & $14 \%$ \\
$z_{t}-z_{w}(\mathrm{~mm})$ & 8.82 & 11.50 & $-30 \%$ \\
$z(l / 2, t)(\mathrm{mm})$ & 10.23 & 9.78 & $4 \%$ \\
$u_{2}(\mathrm{kN})$ & - & 3.60 & - \\
$u_{1}(\mathrm{kN})$ & - & 20.19 & - \\
\hline
\end{tabular}

VI. CONCLUSIONS

A new numerical method for time-varying LQG controller was given and demonstrated by vertical vibration suppressing of a rail carriage traveling on a simply supported uniform girder bridge. Stability and high precision of this algorithm was ensured by combining precise integration method with symplectic conservative perturbation method. Numerical results showed that the vibration of the coupled vehicle-bridge system can be improved to a certain extent by using high-performance suspensions on the vehicle.

\section{ACKNOWLEDGMENT}

Support for this work from the China National Program on Key Basic Research Project (973 Program, grants
2010CB736103 and 2012CB723807), from the International Scientific and Technological Cooperation Project (grant 2011DFA83300) and from the Natural Science Foundation of Chongqing Province of China (grant CSTC2010BB6019) is gratefully acknowledged.

\section{REFERENCES}

[1] H.C. Kwon, M.C. Kim and I.W. Lee, "Vibration control of bridges under moving loads", Computer and Structures, 66, pp. 473-480, 1998.

[2] J.D. Yau and Y.B. Yang, "A wideband MTMD system for reducing the dynamic response of continuous truss bridges to moving train loads", Engineering Structures, 26, pp. 1795-1807, 2004.

[3] J.F. Wang, C.C. Lin and B.L. Chen, "Vibration suppression for high-speed railway bridges using tuned mass dampers", International Journal of Solids and Structures, 40, pp. 465-491, 2003.

[4] M.H. Kargarnovin, D. Younesian, D. Thompson and C. Jones, "Ride comfort of high-speed trains travelling over railway bridges", Vehicle System Dynamics, 43, pp. 173-199, 2005.

[5] Y.S. Wu and Y.B. Yang, "Steady-state response and riding comfort of trains moving over a series of simply supported bridges", Engineering Structures, 25, pp. 251-265, 2003.

[6] J.B. Burl, Linear Optimal Control. Reading, MA: Addison-Wesley, 1999.

[7] C.S. Kenney and R.B. Leipnik, "Numerical integration of the differential matrix Riccati equation", IEEE Transactions on Automatic Control, AC-30, pp. 962-970, 1985.

[8] C.H. Choi, "Time-varying Riccati differential equations with known analytic solutions", IEEE Transactions on Automatic Control, 37, pp. $642-645,1992$.

[9] S.J. Tan and W.X. Zhong, "Numerical solutions of linear quadratic control for time-varying systems via symplectic conservative perturbation", Applied Mathematics and Mechanics, 28, pp. 277-287, 2007.

[10] S.J. Tan and W.X. Zhong, "Numerical solution of differential Riccati equation with variable coefficients via symplectic conservative perturbation method", Journal of Dalian University of Technology, 46, pp. S7-S13, 2006.

[11] W.X. Zhong and Z.Q. Cai, "Precise integration method for LQG optimal measurement feedback control problem", Applied Mathematics and Mechanics, 21, pp. 1417-1422, 2000.

[12] W.X. Zhong, "On precise integration method", Journal of Computational and Applied Mathematics, 163, pp. 59-78, 2004. 